\title{
Optimal Energy Management for Fuel and Emissions Minimization of Series Hybrid Electric Vehicles with Consideration of Engine Preheating
}

\author{
Ruikuan $\mathrm{Hu}^{1}$, Xiao $\mathrm{Pan}^{1}$, Boli $\mathrm{Chen}^{2}$ and Simos A. Evangelou ${ }^{1}$
}

\begin{abstract}
As a result of multiple energy sources, hybrid electric vehicles (HEVs) provide additional flexibility of the engine operating point, which enables optimization of fuel economy and emissions reduction. This paper introduces an energy management (EM) control strategy by an optimal control approach that jointly optimizes fuel consumption and various vehicle pollutant emissions. Engine thermal dynamics are modeled and integrated into the engine-out emission and fuel consumption models for enhanced modeling accuracy. The proposed method is formulated as an optimal control problem (OCP) that is benchmarked against a baseline EM strategy for fuel consumption only optimization. The proposed temperature sensitive emission and fuel consumption models enable a thorough investigation of engine temperature-emissions relationships, which provide important insights into the optimal power split during the engine preheating phase. Simulation results validate the effectiveness of the proposed approach and highlight the importance of analyzing the fuel consumptionemissions trade-off, as small compromises in fuel consumption lead to significant reductions in emissions.
\end{abstract}

\section{INTRODUCTION}

Hybrid electric vehicles (HEVs) represent an important stage for transportation electrification, which has been promoted to address global environmental issues. It is commonly acknowledged that HEVs provide promising fuel efficiency and reduced emissions in comparison with conventional internal combustion engine (ICE) vehicles, due to their capacity of energy recovery and the additional degree of freedom in meeting the driver power demand. It is therefore of great interest to study HEV energy management (EM) control, which involves determining the optimal power allocation between multiple sources in the powertrain. Over the past decade, numerous works have been reported in the literature, with a comprehensive overview of existing EM techniques, from rule-based to optimization-based [1], [2], [3], [4]. In particular, most existing EM strategies are optimizationbased due to optimal or sub-optimal guarantees. The main optimization algorithms encompass dynamic programming (DP) [5], [6], nonlinear programming [7], [8], Pontryagin Minimum Principle (PMP) [9], [10], the equivalent consumption minimization strategy (ECMS) [11], model predictive control (MPC) [12], [13] and machine learning [14].

In most of the existing studies, fuel economy remains the only objective that is optimized whereas the emissions are ignored. It is well acknowledged that fuel-efficient engine operating points do not simultaneously guarantee minimal engine-out emissions [15]. Therefore, the joint consideration of emissions and fuel consumption is an essential step to

\footnotetext{
${ }^{1}$ R. Hu, X. Pan and S. A. Evangelou are with the Dept. of Electrical and Electronic Engineering at Imperial College London, UK (ruikuan.hu19@ic.ac.uk, xiao.pan17@ic.ac.uk, s.evangeloulic.ac.uk)

${ }^{2} \mathrm{~B}$. Chen is with the Dept. of Electronic and Electrical Engineering at University College London, UK (boli.cheneucl.ac.uk)
}

design more practical and potent EM control strategies [16], [17], [18], [19]. In [16], an EM strategy considering the particulate matter (PM) and nitrogen oxide (NOx) emissions is proposed. The strategy is proven to be effective in reducing transient emissions due to the turbocharger lag. A DP-based optimal control strategy is presented in [18] to find the optimal HEV power split that results in minimized fuel consumption and harmful emissions, including NOx, carbon monoxide (CO) and hydrocarbon (HC). The impact of traffic conditions and emissions are integrated into the EM control in [17], where the traffic influence on the driving speed, acceleration, stop and go are predicted to achieve EM control parameters tuning. The very recent work [19] proposes an MPC framework for joint optimization of fuel economy and emissions (CO, HC and NOx) under a car-following scenario.

The production of emissions is highly influenced by the engine thermal behavior, which, however, has not been taken into account in previous studies. An investigation accounting for the engine thermal behavior is particularly important for analyzing optimal power split solutions during the engine preheating phase. The general approach to solve the optimization problem associated with emissions and fuel consumption is based on engine static maps [16], [19] by neglecting the impact of engine temperature changes on the emissions. However, emissions are sensitive to the engine temperature, for example, more NOx is produced at a high engine temperature due to the complex chemical reaction between nitrogen and oxygen at a high temperature, while more $\mathrm{HC}$ and $\mathrm{CO}$ are produced at a low temperature due to the insufficient combustion of fuel in the engine. As such, the accuracy of representing the fuel and emission outputs degrades when the engine temperature is changing dramatically, such as at the preheating stage.

To this end, an optimal control approach to minimize both fuel consumption and various emissions, including NOx, $\mathrm{CO}$ and $\mathrm{HC}$, of a series HEV, with consideration of engine preheating, is proposed in this paper. The main contributions of the paper are therefore as follows: 1) energy management strategy by optimal control framework for simultaneous minimization of fuel consumption and emissions that goes beyond existing strategies by incorporating engine preheating via temperature sensitive fuel consumption and engine-out emission models, 2) investigation of the influence of the engine temperature on the optimal fuel-emissions solutions, 3 ) investigation of the trade-off between fuel consumption and various emissions under the more realistic scenario of engine preheating consideration, and 4) evaluation of the benefit of the joint fuel and emissions optimization framework by comparisons with a baseline fuel-only optimization EM strategy in most existing EM control strategies [1], [3].

This paper is organized as follows. Section II introduces the powertrain and emissions models of a series HEV. 
Section III describes the proposed EM strategy and baseline strategy. Section IV presents numerical examples and comparisons, followed by the concluding remarks in Section V.

\section{Model Description}

\section{A. Series Hybrid Electric Vehicle Powertrain Model}

The series HEV powertrain is sketched in Fig. 1. As it

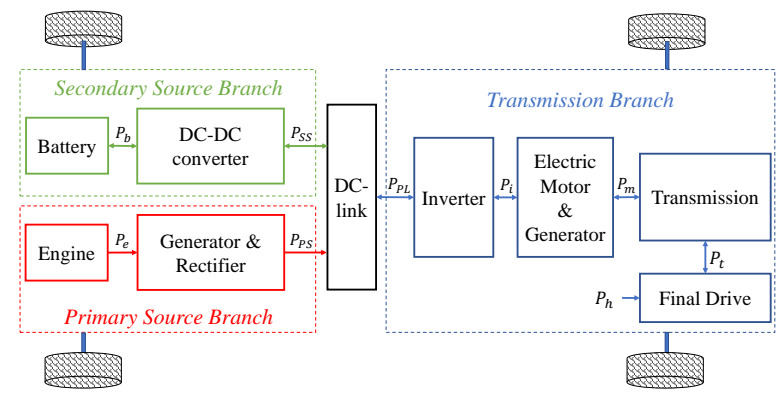

Fig. 1. Powertrain configuration for series hybrid electric vehicles.

can be seen, the series powertrain consists of two energy branches: the primary source (PS) branch and the secondary source (SS) branch, which are jointed at a DC-link subject to the power balance equation:

$$
P_{P L}=P_{P S}+P_{S S},
$$

where $P_{P S}$ and $P_{S S}$ are power outputs of the PS and SS branches. The combined electric power $P_{P L}$ is delivered to the driving wheels via the transmission branch that also includes an electric motor. When the vehicle is decelerating, braking energy can be regenerated by operating the motor as a generator. Mechanical brakes can provide additional braking power, which is converted into heat and dissipated.

The main characteristic parameters of the vehicle model are listed in Table. I, which are chosen based on a mediumsized passenger car with a non plug-in hybrid powertrain.

1) Primary Source Branch: The PS branch contains an ICE, a permanent magnet synchronous generator (PMSG), and a rectifier, which are connected in series. The ICE in the present work is a 2.0L Saab gasoline engine [15]. The output power of the PS branch is given by:

$$
P_{P S}=\eta_{r} \eta_{g} P_{e}\left(\tau_{e n g}, \omega_{e n g}\right),
$$

where $\eta_{g}$ and $\eta_{r}$ are constant efficiencies of the generator and rectifier, and $P_{e}$ is the engine output power for the engine torque $\tau_{e n g}$ and the crankshaft speed $\omega_{e n g}$. The dynamics of the engine fuel consumption mass $m_{f}$ and engine-out emissions masses $m_{N O x}, m_{C O}, m_{H C}$ for NOx, CO and $\mathrm{HC}$ emissions, will be introduced in the next section.

2) Secondary Source Branch: The SS branch contains a battery and a DC-DC converter, which allows the battery to be charged and discharged via bidirectional power flow. The battery in this work is modeled as a series connection of an ideal voltage source and a resistance [20]. Furthermore, the SS output power is obtained by:

$$
P_{S S}=\eta_{d c}^{\operatorname{sign}\left(P_{S S}\right)} P_{b},
$$

where $\eta_{d c}$ is the efficiency for DC-DC converter and $P_{b}$ is the battery output power. In this modeling framework, the battery state of charge (SoC) is the only dynamic state of
TABLE I

Main Vehicle MODEl PARAMETERS

\begin{tabular}{lll}
\hline \hline Description & Symbol & Value \\
\hline Vehicle mass & $m$ & $1380 \mathrm{~kg}$ \\
Wheel radius & $r_{w}$ & $0.3 \mathrm{~m}$ \\
Air drag coefficient & $f_{d}$ & 0.47 \\
Tyre rolling coefficient & $f_{t}$ & 0.01 \\
Conversion Efficiencies & $\eta_{i}, \eta_{t}, \eta_{g}, \eta_{r}$ & 0.96 \\
Battery power limits & $P_{b, \max } / \mathrm{min}$ & $27 /-16.5 \mathrm{~kW}$ \\
Battery open circuit voltage & $V_{O C}$ & $300 \mathrm{~V}$ \\
Battery maximum capacity & $Q_{\max }$ & $15 \mathrm{Ah}$ \\
Battery resistance & $R_{b}$ & $0.2056 \Omega$ \\
Battery SoC limits & $\mathrm{SoC}_{\mathrm{max}} / \mathrm{min}$ & $0.8 / 0.5$ \\
Gasoline lower heating value & $Q_{H V}$ & $44 \mathrm{~kJ} / \mathrm{g}$ \\
Equivalent engine efficiency & $\alpha_{f}$ & 0.255 \\
Ave. ICE heat discharging rate & $P_{d i s}$ & $1 \mathrm{~kW}$ \\
ICE preheating energy & $Q_{r e q}$ & $892.5 \mathrm{~kJ}$ \\
Fuel thermal-weighting factor & $\xi_{f}$ & 1.85 \\
NOx thermal-weighting factor & $\xi_{N O_{x}}$ & 0.35 \\
CO thermal-weighting factor & $\xi_{C O}$ & 9.83 \\
HC thermal-weighting factor & $\xi_{H C}$ & 12.39 \\
Fuel normalizing factor & $m_{f, \text { scale }}$ & $2000 \mathrm{~g}$ \\
NOx normalizing factor & $m_{N O x}$, scale & $100 \mathrm{~g}$ \\
HC normalizing factor & $m_{H C, \text { scale }}$ & $100 \mathrm{~g}$ \\
CO normalizing factor & $m_{C O, \text { scale }}$ & $400 \mathrm{~g}$ \\
\hline \hline
\end{tabular}

the battery system, and its dynamics are governed by:

$$
\frac{d}{d t} \mathrm{SoC}=-\frac{V_{O C}+\sqrt{V_{O C}^{2}-4 R_{b} P_{S S} / \eta_{d c}^{\operatorname{sign}\left(P_{S S}\right)}}}{2 R_{b} Q_{\max }},
$$

where $V_{O C}$ is the battery open circuit voltage, $R_{b}$ is the battery resistance, and $Q_{\max }$ is the battery maximum capacity. The operation of the battery SoC is constrained by $\left[\mathrm{SoC}_{\min }, \mathrm{SoC}_{\text {max }}\right]$.

3) Transmission Branch: From the flow within the transmission branch, the relationship between $P_{P L}$ and the output power of the powertrain $P_{t}$ is expressed as:

$$
P_{t}=\left(\eta_{i} \eta_{m} \eta_{t}\right)^{\operatorname{sign}\left(P_{t}\right)} P_{P L}
$$

where $\eta_{i}$ and $\eta_{t}$ are constant efficiencies of the inverter and the transmission. $\eta_{m}$ is the efficiency of the bidirectional motor/generator represented by an efficiency map for varying motor load torque and angular speed, as shown in Fig. 2. With consideration of the mechanical braking power $P_{h}<0$,

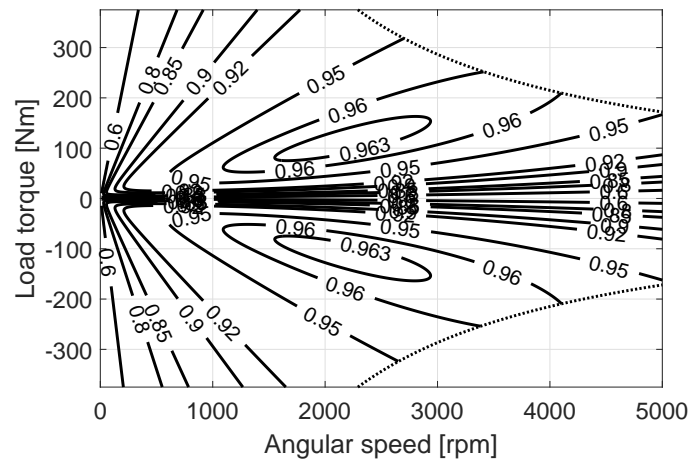

Fig. 2. Efficiency map of the reversible Permanent Magnet Synchronous (PMS) machine (positive torque $=$ generator, negative torque $=$ motor) . Dotted lines present the torque bounds due to power limitation [20].

the driving power $P_{v}$ that acts on the wheels is:

$$
P_{v}=P_{t}+P_{h} .
$$




\section{B. Fuel Consumption and Emission Models}

In this subsection, the dynamics of ICE temperature are modeled, and the fuel consumption and emission models, which are functions of ICE temperature, are developed.

1) Engine Temperature Model: The engine temperature index $I_{t e m}$ is introduced to indicate the percentage of the engine temperature, where $I_{t e m}=0$ represents the cold engine condition at an ambient temperature $T_{a m b}$, and $I_{t e m}=$ 1 represents the steady state temperature $T_{s s}$ engine works at. The dynamic equation of $I_{t e m}$ is given as:

$$
\frac{d}{d t} I_{t e m}=\frac{\dot{m}_{f} Q_{H V}\left(1-\alpha_{f}\right)-P_{d i s}}{Q_{r e q}},
$$

where $\dot{m}_{f}$ is the instantaneous fuel rate, $Q_{H V}$ is the gasoline lower heating value, $\alpha_{f}$ is the equivalent fuel efficiency, $P_{d i s}$ is the average heat discharging rate by engine cooling and exhaust waste gas, and $Q_{r e q}$ is the estimated total heat demand for preheating, which is calculated by:

$$
Q_{r e q}=\left(T_{s s}-T_{a m b}\right) \beta,
$$

where $T_{s s}=105^{\circ} \mathrm{C}$ is the engine working temperature, $T_{a m b}=20^{\circ} \mathrm{C}$ is the nominal cold temperature, and $\beta=10.5$ $\mathrm{kJ} / \mathrm{K}$ is an empirical conversion coefficient [15].

2) Fuel Consumption Model: In the context of series HEVs, conventional EM strategies targeting optimal fuel economy simply assume the ICE to be operated at optimal efficiency points, with the consequence that the fuel mass rate relationship to the PS output power can be approximated as a linear function [10], [13]. However, the high-efficient region for fuel consumption may not necessarily be equivalent to the minimum emissions region. Thus, the dynamics of the engine fuel mass consumption in this work is described as a nonlinear function of the engine torque $\tau_{e n g}$ and the crankshaft speed $\omega_{\text {eng }}$, as with a parallel hybrid electric or a conventional vehicle [15]:

$$
\dot{m}_{f}=\dot{m}_{f, s s}\left(\tau_{e n g}, \omega_{e n g}\right)\left[I_{t e m}+\xi_{f}\left(1-I_{t e m}\right)\right],
$$

where $\dot{m}_{f}$ represents the instantaneous fuel mass rate, $\dot{m}_{f, s s}\left(\tau_{e n g}, \omega_{e n g}\right)$ is the fuel mass rate obtained from the ICE static map (see Fig. 3), and $\xi_{f}$ is assumed to be a constant ratio representing the cold start and fully hot fuel mass rate and it is estimated by fitting cold-start data of the Saab engine with equation (9).

3) Engine-out Emission Model: Similarly, the dynamics of emissions mass rate are described by [15]:

$$
\dot{m}_{i}=\dot{m}_{i, s s}\left(\tau_{e n g}, \omega_{e n g}\right)\left[I_{t e m}+\xi_{i}\left(1-I_{t e m}\right)\right],
$$

where $\dot{m}_{i}$ is the instantaneous mass rate for emission $i$ with $i \in\{\mathrm{NOx}, \mathrm{CO}, \mathrm{HC}\}, \dot{m}_{i, s s}\left(\tau_{\text {eng }}, \omega_{\text {eng }}\right)$ is the mass rate of emission $i$ obtained from the static maps respectively for the three emissions (see Fig. 3), and $\xi_{i}$ is assumed to be a constant ratio representing the cold start and fully hot emission mass rate of species $i$ and it is estimated by fitting cold-start data of the Saab engine with equation (10).

\section{Optimization PROBLEM Formulation}

This section introduces the EM control strategy for series HEVs to optimize the fuel economy and the engine-out emissions. The EM optimization strategy finds the SS branch power $P_{S S}$, the engine torque $\tau_{e n g}$ and the engine crankshaft
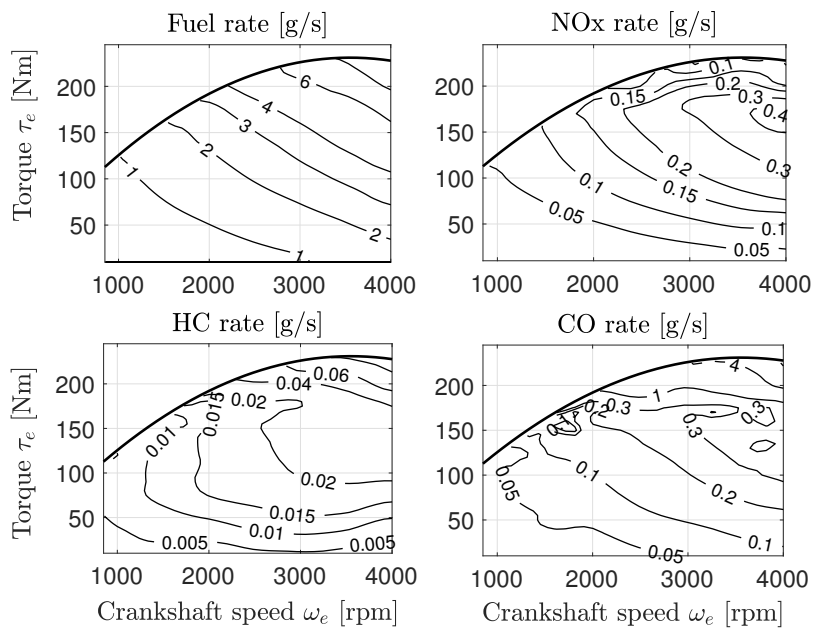

Fig. 3. Fuel mass rate and emission rate maps with respect to engine torque $T_{e}$ and crankshaft speed $\omega_{e}$ for a $2.0 \mathrm{~L}$ Saab gasoline engine at hot steady state [15].

speed $\omega_{\text {eng }}$ over a specified time-horizon $[0, T]$ for a given driving cycle, such that both the fuel consumption $m_{f}(T)$ and the emissions $m_{i}(T)$ can be co-optimized and the battery is charge-sustained at the end of the mission, subject to the ICE and battery operational constraints. Before introducing the novel scheme, a baseline strategy for benchmarking purposes is introduced first.

\section{A. Fuel-only Optimization Strategy (Baseline Method)}

The fuel-only optimization strategy utilizes the general power-split optimization framework to minimize fuel consumption. The objective function is given as:

$$
J_{\text {fuel }}=\int_{0}^{T} \dot{m}_{f} d t
$$

where $\dot{m}_{f}$ is the only term in the objective function, and the optimal solutions of (11) can be set as a baseline for comparing the performance of the joint optimization on both fuel consumption and emissions.

\section{B. Joint Emission Optimization Strategy}

In contrast to the baseline formulation, the proposed EM strategy is obtained by minimizing the following miltobjective cost function:

$$
J_{\text {joint }}=\int_{0}^{T} W_{\text {fuel }} \frac{\dot{m}_{f}}{m_{f, \text { scale }}}+\sum_{i} W_{i} \frac{\dot{m}_{i}}{m_{i, \text { scale }}} d t,
$$

where $i \in\{\mathrm{NOx}, \mathrm{CO}, \mathrm{HC}\}, W_{\text {fuel }}+\sum_{i} W_{i}=1, m_{f}$, scale and $m_{i, s c a l e}$ are constant given in Table. I so that the individual costs are normalized, and $W_{\text {fuel }}$ and $W_{i}$ are weighting factors of fuel and emissions, respectively.

\section{Tuning of Weighting Factors}

One of the fundamental challenges for multi-objective optimization is the weight tuning. In order to find the effective trade-off between the four objectives with minimum tuning of the weights, pairwise interactions between fuel and emissions costs are first investigated. In this context, the following sub-optimization problems are formulated: 1) 
minimizing the weighted sum of fuel consumption and one of the emissions:

$$
J_{\text {partial }_{1}}=\int_{0}^{T} W_{\text {fuel }} \frac{\dot{m}_{f}}{m_{f, \text { scale }}}+W_{i} \frac{\dot{m}_{i}}{m_{i, \text { scale }}} d t,
$$

where $i \in\{\mathrm{NOx}, \mathrm{CO}, \mathrm{HC}\}$ and $W_{\text {fuel }}+W_{i}=1$; and 2 ) minimizing the weighted sum of two emissions:

$$
J_{\text {partial }_{2}}=\int_{0}^{T} W_{i} \frac{\dot{m}_{i}}{m_{i, \text { scale }}}+W_{j} \frac{\dot{m}_{j}}{m_{j, \text { scale }}} d t,
$$

where $i, j \in\{\mathrm{NOx}, \mathrm{CO}, \mathrm{HC}\}$ with $i \neq j$ and $W_{i}+W_{j}=1$. For both cases, $m_{f, \text { scale }}, m_{i, \text { scale }}, m_{j \text {, scale }}$ are normalizing factors, given in Table. I and $W_{f u e l}, W_{i}, W_{j}$ are constant weights tuned to balance the control performance.

\section{Optimal Control Problem Formulation}

For a given driving cycle in terms of speed $v$ and acceleration $a$, the driving force acting on the wheels $F_{v}$ can be calculated as:

$$
F_{v}=m a+f_{T} m g+f_{D} v^{2}+m g \sin \theta,
$$

where $m$ is the vehicle mass, $f_{T}$ and $f_{D}$ are the coefficients of tyre rolling and aerodynamic drag resistance, respectively, and $\theta$ is the road slope. As such, the load power demand $P_{P L}$ at the DC-link can be calculated directly from the power at the wheels, $P_{v}=F_{v} v$, as follows [20]:

$$
\begin{cases}P_{P L}=\frac{P_{v}}{\eta_{i} \eta_{m} \eta_{t}}, & \forall P_{v} \geq 0, \\ P_{P L}=\left(P_{v}-P_{h}\right) \eta_{i} \eta_{m} \eta_{t}, & \forall P_{v}<0 .\end{cases}
$$

where $P_{h}$ in this context is calculated by: $P_{h}=\min \left\{0, P_{v}-\right.$ $\left.P_{S S, \min /\left(\eta_{i} \eta_{m} \eta_{t}\right)}\right\}$.

Thus, to find the optimal power-split provided by the primary and secondary sources which minimize both fuel consumption and emissions, the OCP is formulated as:

$$
\begin{array}{ll} 
& \min _{\mathbf{u}} J \\
\text { s.t.: } & \frac{d}{d t} \mathbf{x}=f(\mathbf{x}, \mathbf{u}), \\
& \Psi(\mathbf{x}, \mathbf{u}) \leq 0 \\
& \boldsymbol{b}(\mathbf{x}(0), \mathbf{x}(L))=0
\end{array}
$$

where $J \in\left\{J_{\text {fuel }}, J_{\text {partial }_{1}}, J_{\text {partial }_{2}}, J_{\text {joint }}\right\}$, the state variable is $\mathbf{x} \triangleq\left[\mathrm{SoC}, I_{t e m}, m_{f}, m_{i}\right]^{\top}$, the control variable is $\mathbf{u} \triangleq\left[P_{s s}, \tau_{\text {eng }}, \omega_{\text {eng }}\right]^{\top}$, and the state space model $\frac{d}{d t} \mathbf{x}=$ $f(\mathbf{x}, \mathbf{u})$ is described as:

$$
\frac{d}{d t} \mathbf{x}=\left(\begin{array}{c}
\frac{-V_{O C}+\sqrt{V_{O C}^{2}-4 R_{b} P_{S S} / \eta_{d c}^{\operatorname{sign}\left(P_{S S}\right)}}}{2 R_{b} Q_{\text {max }}} \\
\frac{\dot{m}_{f} Q_{H V}\left(1-\alpha_{f}\right)-P_{d i s}}{Q_{r e q}} \\
\dot{m}_{f, s s}\left(\tau_{e n g}, \omega_{e n g}\right)\left(I_{t e m}+\xi_{f}\left(1-I_{t e m}\right)\right) \\
\dot{m}_{i, s s}\left(\tau_{e n g}, \omega_{e n g}\right)\left(I_{t e m}+\xi_{i}\left(1-I_{t e m}\right)\right)
\end{array}\right)
$$

with $i \in\{\mathrm{NOx}, \mathrm{CO}, \mathrm{HC}\}$. The inequality constraint (17c) is imposed due to physical and operation limits:

$$
\begin{aligned}
& 0 \leq P_{P S} \leq P_{P S_{\max }}, \quad P_{S S_{\min }} \leq P_{S S} \leq P_{S S_{\max }} \\
& \mathrm{SoC}_{\min } \leq \mathrm{SoC} \leq \mathrm{SoC}_{\max }, \quad 0 \leq I_{\text {tem }} \leq 1, \\
& P_{P S}+P_{S S} \geq P_{P L},
\end{aligned}
$$

where (19c) is designed to relax the equality constraint (1) since the fuel consumption is minimized and the battery aims to achieve charge sustaining, both $P_{P S}$ and $P_{S S}$ find their minimum boundaries as solutions. The problem is completed by the boundary conditions (17d), expressed as follows:

$$
\begin{aligned}
& \operatorname{SoC}(0)=0.65, \quad \operatorname{SoC}(T)=0.65 \\
& I_{\text {tem }}(0)=0, \quad m_{f}(0)=0, \\
& m_{N O_{x}}(0)=0, \quad m_{C O}(0)=0, \quad m_{H C}(0)=0 .
\end{aligned}
$$

\section{NumericAl Results}

The performance of the proposed scheme is evaluated in this Section and its solution is compared with the solution of the baseline strategy described in Section III. The driving cycle defines the speed profile that needs to be followed by the HEVs. Without loss of generality, the first three stages of the worldwide harmonized light vehicles test cycles (WLTP) cycle (excluding the extra high) are selected, to emulate non-highway driving. As shown in Fig. 4, the speed profile utilized in this work is formed by three individual profiles, separated based on their average speeds: low (WLL), medium (WL-M) and high (WL-H). By using (16), it is immediate to determine the PL power demand as illustrated in Fig. 4. The numerical evaluation of the proposed EM

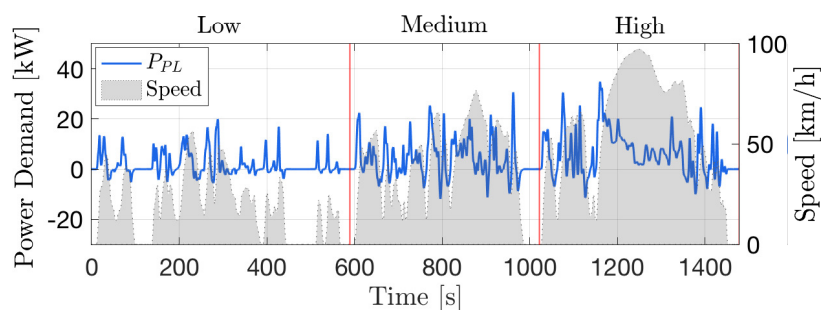

Fig. 4. Speed profile of the WLTP with three different stages and the associated power demand $P_{P L}$ obtained by (16).

method is performed in two steps:

1) A thorough investigation of pairwise relationships between fuel cost and each emission by using the objective functions (13) and (14) is conducted. The results provide useful insight into the weighting of the proposed fourfold joint optimization in (12).

2) With the weights found in step 1), the benefits of the joint optimization in terms of the fuel consumption and emissions are illustrated by comparisons with the baseline method, and the influence of the engine temperature is analyzed.

All the optimization problems are solved by GPOPS-II [21] in the Matlab environment on a personal computer with Intel Core i5 $1.8 \mathrm{GHz}$ and $8 \mathrm{~GB}$ of RAM.

Some insightful results for the tuning of the weights are reported in Fig. 5. As it can seen, a monotonically decreasing relationship between fuel consumption and NOx outputs is illustrated in Fig. 5(a). Similarly, a negative relationship also holds between NOx and $\mathrm{CO}$, and and between NOx and HC as shown in Figs. 5(b) and 5(c), respectively. Conversely, the relationship between $\mathrm{HC}$ and $\mathrm{CO}$ is positive as indicated in Fig. 5(d). Based on the above analysis, the objective function 


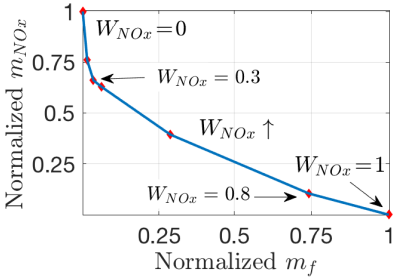

(a)

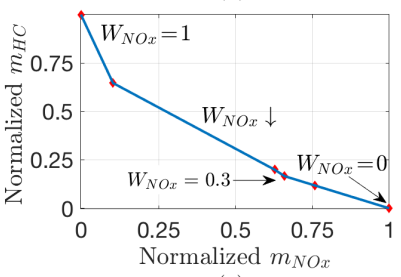

(c)

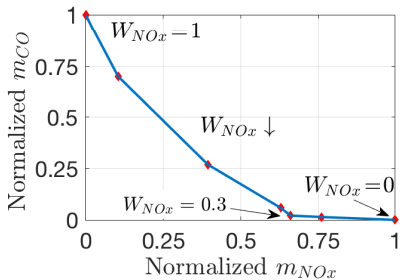

(b)

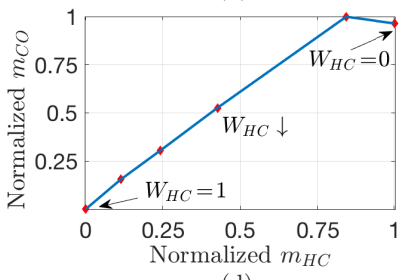

(d)
Fig. 5. Pairwise trade-offs between fuel and emissions (a) fuel consumption and NOx; (b) CO and NOx; (c) HC and NOx; (d) CO and HC. All the emissions and fuel consumption are normalized.

of the proposed method (12) can be rewritten as:

$$
\begin{array}{r}
J_{\text {joint }}=\int_{0}^{T} W_{\text {fuel }} \frac{\dot{m}_{f}}{m_{f, \text { scale }}}+W_{N O x} \frac{\dot{m}_{N O x}}{m_{N O X, \text { scale }}}+ \\
W_{H C}\left(\frac{\dot{m}_{H C}}{m_{H C, \text { scale }}}+\frac{\dot{m}_{C O}}{m_{C O, \text { scale }}}\right) d t
\end{array}
$$

where $W_{f u e l}+W_{N O x}+2 W_{H C}=1$ and $W_{H C}=W_{C O}$. Moreover, the optimal solutions in Fig. 5(a) show that when $W_{N O x}$ increases from 0 to 0.3 , it yields a $37 \%$ reduction in $m_{N O x}$ with only $6.1 \%$ increase in $m_{f}$. However, further increasing $W_{\text {fuel }}$ from 0.3 will lead to a significant increase in fuel cost. As such, $W_{N O x}=0.3$ is a proper choice in terms of the trade-off between NOx and fuel cost, as a small compromise in fuel cost leads to a significant reduction in NOx. As with the fuel consumption, $\mathrm{HC}$ and $\mathrm{CO}$ also have a negative relationship with NOx, and $W_{N O x}=0.3$ remains a proper selection yielding effective trade-offs between the NOx and other emissions. Therefore, it is appropriate to set $W_{N O x}=0.3$ and $W_{\text {fuel }}+2 W_{H C}=0.7$ for $(21)$.

Next, the proposed joint OCP is solved for a set of weights (see Table II) based on the previously identified values, and the solutions are benchmarked against the baseline solution (see Table III and Fig. 6). Compared to the baseline method,

TABLE II

DESIGN OF WEIGHTING FACTORS FOR THE PROPOSED APPROACH (21)

\begin{tabular}{cccc}
\hline \hline Strategy & $W_{\text {fuel }}$ & $W_{\text {NOx }}$ & $W_{H C}$ \\
\hline Joint-1 & 0.1 & 0.3 & 0.3 \\
Joint-2 & 0.2 & 0.3 & 0.25 \\
Joint-3 & 0.3 & 0.3 & 0.2 \\
\hline \hline
\end{tabular}

TABLE III

FUEL CONSUMPTION AND EMISSIONS OF JOINT OPTIMIZTION (21) COMPARED WITH BASELINE METHOD (11)

\begin{tabular}{lcccc}
\hline Strategy & $m_{f}[\mathrm{~g}]$ & $m_{N O x}[\mathrm{~g}]$ & $m_{H C}[\mathrm{~g}]$ & $m_{C O}[\mathrm{~g}]$ \\
\hline Baseline & 669.2 & 16.1 & 13.1 & 58.8 \\
\hline Joint-1 & $679.2(+1.5 \%)$ & $15.6(-3.5 \%)$ & $12.3(-6.1 \%)$ & $50.5(-14.1 \%)$ \\
Joint-2 & $676.3(+1.1 \%)$ & $15.5(-3.8 \%)$ & $12.2(-7 \%)$ & $50.9(-13.4 \%)$ \\
Joint-3 & $675.1(+0.9 \%)$ & $15.5(-3.6 \%)$ & $12.8(-2.8 \%)$ & $55(-6.4 \%)$ \\
\hline \hline
\end{tabular}

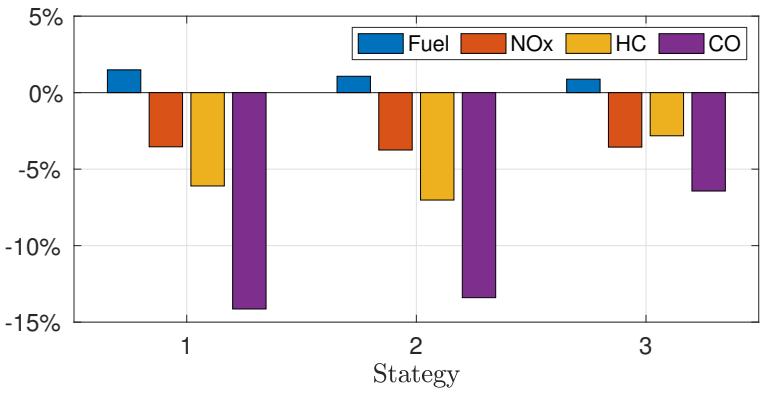

Fig. 6. Optimality of the proposed joint optimization strategy compared with the baseline solution in terms of fuel consumption and emissions.

all the joint optimization strategies can significantly reduce the emissions with marginal fuel increase. For example, the joint optimization strategy 2 with $W_{\text {fuel }}=0.2, W_{N O x}=0.3$, $W_{H C}=W_{C O}=0.25$ can reduce all three types of emissions by $3.75 \%$ of NOx, $7.02 \%$ of $\mathrm{HC}$, and $13.40 \%$ of $\mathrm{CO}$ as compared to the baseline solution, with $1.07 \%$ fuel increase.

Then, the influence of the engine temperature on the optimal fuel and emission solutions is investigated. With the weighting factors set to $W_{\text {fuel }}=0.2, W_{N O x}=0.3$, $W_{H C}=0.25$, the engine temperature profile of the joint optimization solution is compared with that of the baseline solution in Fig. 7. As it can be seen, although the ICE

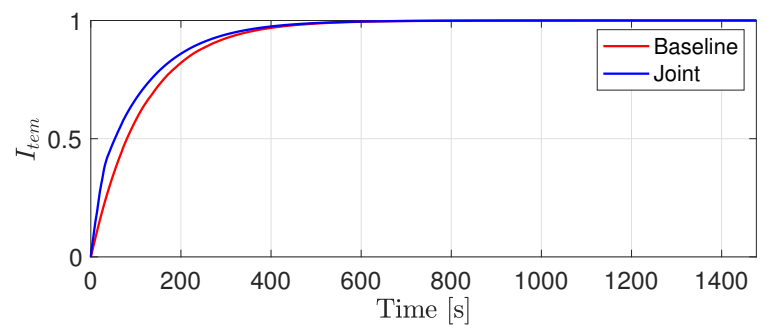

Fig. 7. Engine temperature index $I_{\text {tem }}$ of the proposed joint optimization and the baseline solution.

temperatures $I_{t e m}$ of both strategies can reach the working condition $I_{t e m}=1$ at the steady state, the proposed method tends to speed up the preheating process leading to a faster temperature convergence with a settling time of $324 \mathrm{~s}, 6.4 \%$ earlier than the baseline solution.

The comparison in terms of fuel consumption and emissions shown in Fig. 8 further implies that the faster preheating of the joint optimization methods can significantly cut $\mathrm{CO}$ and $\mathrm{HC}$ emissions, as their mass rates are extremely high when the engine is cold (before $300 \mathrm{~s}$ ) but both $\mathrm{HC}$ and $\mathrm{CO}$ are less than the baseline much before 300 s due to the fact that $\xi_{C O}, \xi_{H C} \gg 1$ [15]. At the same time, relatively higher engine temperature for the joint optimization case yields more NOx, as the mass rate of NOx increases as the $I_{t e m}$ increases (as a result of $\xi_{N O x} \ll 1$ ). Despite the joint optimization solution produces more NOx than the baseline solution during the preheating phase, the joint optimization produces fewer NOx emissions as compared to the baseline strategy when the ICE temperature in both cases reaches the working condition as emission is co-optimized. Similar behavior can be observed in the fuel usage profile. Moreover, the peak at about 60s indicates when the fuel mass rate of the joint optimization method becomes smaller than the baseline strategy. This is also the time when the temperature 


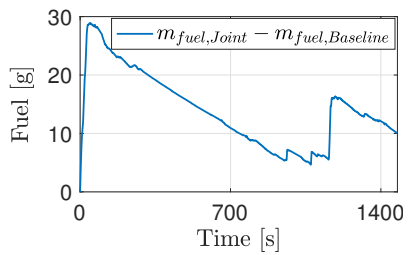

(a)

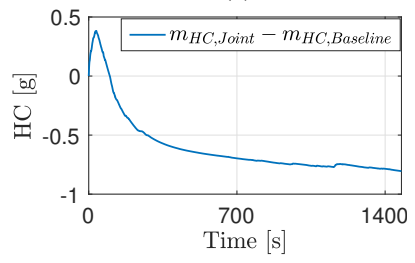

(c)

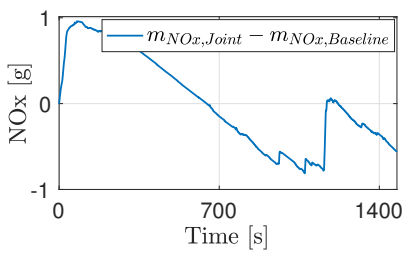

(b)

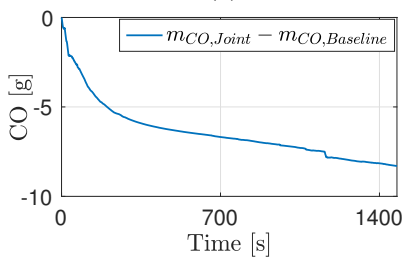

(d)
Fig. 8. Fuel and emissions mass differences of the proposed energy management strategy against the baseline method. (a) Fuel, (b) Nox, (c) $\mathrm{HC}$, and (d) CO.

difference between the two methods is the highest, as shown in Fig. 7. The SoC profiles shown in Fig. 9 indicate that the additional fuel mass rate before 60 s requested by the joint optimization strategy is intended to increase the temperature of the engine, while charging the battery so that less engine power is required in the remainder of the mission. As such, the proposed approach is able to reduce all three emissions (CO, NOx and HC) with a marginal fuel increase.

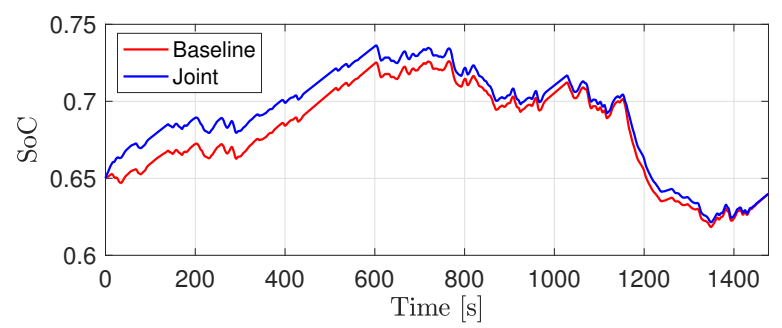

Fig. 9. Battery state of charge of the proposed joint optimization and baseline solution.

\section{CONCLUSION}

This paper proposes an optimal EM strategy for series HEVs to minimize both fuel consumption and emissions (NOx, HC and $\mathrm{CO}$ ) with consideration of engine preheating. An important step for designing the fuel-emissions joint optimization framework is to identify the correlation between these costs. This paper studies the pairwise trade-off between fuel and each emission, which yields the weights of the joint optimization. By modeling the engine temperature, the relationships between fuel, emissions and temperature in the optimization results are examined. In contrast with the fuelonly optimization approach, the proposed joint EM method encourages faster engine preheating, which can significantly reduce emissions with a small compromise in fuel consumption. A representative example shows that the proposed joint optimization strategy is able to reduce $3.75 \%$ of NOx, $7.02 \%$ of $\mathrm{HC}$, and $13.40 \%$ of $\mathrm{CO}$, at a price of $1.07 \%$ more fuel usage as compared with the baseline method.

Further studies focus on the integration of additional modeling details, such as the engine start-stop system, and realtime implementation (e.g. nonlinear model predictive control
(NMPC) techniques or data-driven optimization methods). Moreover, robust optimization schemes will be developed to cope with the uncertainty in model and decision parameters.

\section{REFERENCES}

[1] F. Zhang, X. Hu, and D. Cao, "Energy management strategies of connected HEVs and PHEVs: Recent progress and outlook," Progress in Energy and Combustion Science, vol. 73, pp. 235-256, 2019.

[2] W. Shabbir and S. A. Evangelou, "Threshold-changing control strategy for series hybrid electric vehicles," Applied Energy, vol. 235, pp. 761775, 2019.

[3] C. M. Martinez, X. Hu, D. Cao, E. Velenis, B. Gao, and M. Wellers, "Energy management in plug-in hybrid electric vehicles: Recent progress and a connected vehicles perspective," IEEE Transactions on Vehicular Technology, vol. 66, no. 6, pp. 4534-4549, 2017.

[4] A. A. Malikopoulos, "Supervisory power management control algorithms for hybrid electric vehicles: A survey," IEEE Transactions on intelligent transportation systems, vol. 15, no. 5, pp. 1869-1885, 2014.

[5] L. V. Pérez and E. A. Pilotta, "Optimal power split in a hybrid electric vehicle using direct transcription of an optimal control problem," Mathematics and Computers in Simulation, vol. 79, no. 6, pp. 19591970, 2009.

[6] Y. L. Murphey, J. Park, L. Kiliaris, M. L. Kuang, M. A. Masrur, A. M. Phillips, and Q. Wang, "Intelligent hybrid vehicle power controlpart II: Online intelligent energy management," IEEE Transactions on Vehicular Technology, vol. 62, no. 1, pp. 69-79, 2012.

[7] B. Chen, S. A. Evangelou, and R. Lot, "Hybrid electric vehicle twostep fuel efficiency optimization with decoupled energy management and speed control," IEEE/ASME Transactions on Vehicular Technology, vol. 68, no. 12, pp. 11492-11 504, 2018.

[8] S. Uebel, N. Murgovski, C. Tempelhahn, and B. Baker, "Optimal energy management and velocity control of hybrid electric vehicles,' IEEE Transactions On Vehicular Technology, vol. 67, no. 1, pp. 327337, 2018.

[9] C. Hou, M. Ouyang, L. Xu, and H. Wang, "Approximate pontryagins minimum principle applied to the energy management of plug-in hybrid electric vehicles," Applied Energy, vol. 115, pp. 174-189, 2014.

[10] B. Chen, S. A. Evangelou, and R. Lot, "Series hybrid electric vehicle simultaneous energy management and driving speed optimization," IEEE/ASME Transactions on Mechatronics, vol. 24, no. 6, pp. 27562767, 2018.

[11] L. Serrao, S. Onori, and G. Rizzoni, "A comparative analysis of energy management strategies for hybrid electric vehicles," Journal of Dynamic Systems, Measurement, and Control, vol. 133, no. 3, 2011.

[12] J. Zhang and T. Shen, "Real-time fuel economy optimization with nonlinear MPC for PHEVs," IEEE Transactions on Control Systems Technology, vol. 24, no. 6, pp. 2167-2175, 2016.

[13] X. Pan, B. B. Chen, and S. Evangelou, "Optimal vehicle following strategy for joint velocity and energy management control of series hybrid electric vehicles," in 21st IFAC World Congress, 2020.

[14] X. Hu, T. Liu, X. Qi, and M. Barth, "Reinforcement learning for hybrid and plug-in hybrid electric vehicle energy management: Recent advances and prospects," IEEE Industrial Electronics Magazine, vol. 13 , no. 3 , pp. 16-25, 2019.

[15] Z. Gao, J. Conklin, C. S. Daw, and V. K. Chakravarthy, "A proposed methodology for estimating transient engine-out temperature and emissions from steady-state maps," International Journal of Engine Research, vol. 11, no. 2, pp. 137-151, 2010.

[16] T. Nüesch, M. Wang, P. Isenegger, C. H. Onder, R. Steiner, P. MacriLassus, and L. Guzzella, "Optimal energy management for a diesel hybrid electric vehicle considering transient PM and quasi-static NOx emissions," Control Engineering Practice, vol. 29, pp. 266-276, 2014.

[17] M. Montazeri-Gh and M. Mahmoodi-K, "Optimized predictive energy management of plug-in hybrid electric vehicle based on traffic condition," Journal of cleaner production, vol. 139, pp. 935-948, 2016.

[18] J. Knapp, A. Chapman, S. Mody, and T. Steffen, "Optimal control inputs for fuel economy and emissions of a series hybrid electric vehicle," SAE Technical Paper, Tech. Rep., 2015.

[19] X. Hu, X. Zhang, X. Tang, and X. Lin, "Model predictive control of hybrid electric vehicles for fuel economy, emission reductions, and inter-vehicle safety in car-following scenarios," Energy, vol. 196, p. $117101,2020$.

[20] B. Chen, X. Li, S. A. Evangelou, and R. Lot, "Joint propulsion and cooling energy management of hybrid electric vehicles by optimal control," IEEE Transactions on Vehicular Technology, vol. 69, no. 5, pp. 4894-4906, 2020.

[21] M. A. Patterson and A. V. Rao, "GPOPS - II: A MATLAB software for solving multiple-phase optimal control problems using hpadaptive gaussian quadrature collocation methods and sparse nonlinear programming," ACM Transactions on Mathematical Software, 2014. 